PROCEEDINGS OF THE

AMERICAN MATHEMATICAL SOCIETY

Volume 135, Number 8, August 2007, Pages 2665-2667

S 0002-9939(07)08812-0

Article electronically published on February 9, 2007

\title{
POLYNOMIAL MAPS AND EVEN DIMENSIONAL SPHERES
}

\author{
FRANCISCO-JAVIER TURIEL \\ (Communicated by Paul Goerss)
}

\begin{abstract}
We construct, for every even dimensional sphere $S^{n}, n \geq 2$, and every odd integer $k$, a homogeneous polynomial map $f: S^{n} \rightarrow S^{n}$ of Brouwer degree $k$ and algebraic degree $2|k|-1$.
\end{abstract}

A polynomial map from $X \subset \mathbb{R}^{m}$ to $Y \subset \mathbb{R}^{r}$ is the restriction to $X$ of a polynomial map $F: \mathbb{R}^{m} \rightarrow \mathbb{R}^{r}$ such that $F(X) \subset Y$. When each component of $F$ is homogeneous of degree $k$, we will say that the polynomial map from $X \subset \mathbb{R}^{m}$ to $Y \subset \mathbb{R}^{r}$ is homogeneous of degree $k$. As usual $S^{n}$ is the sphere in $\mathbb{R}^{n+1}$ defined by the equation $x_{1}^{2}+\ldots+x_{n+1}^{2}=1$, in short $\|x\|^{2}=1$, whereas $S_{r}^{n}, 1 \leq r \leq n$, will be the differentiable manifold, diffeomorphic to $S^{n}$, defined by the equation $\left(x_{1}^{2}+\ldots+x_{r}^{2}\right)^{2}+x_{r+1}^{2}+\ldots+x_{n+1}^{2}=1$. In this work we show:

Theorem 1. Suppose $n$ even and $\geq 2$. Let $k$ be an integer. Then:

(a) If $k$ is odd, there exists a homogeneous polynomial map from $S^{n}$ to $S^{n}$ of Brouwer degree $k$ and algebraic degree $2|k|-1$.

(b) If $k$ is even there exists, for each $2 \leq 2 r \leq n$, a polynomial map from $S_{2 r}^{n}$ to $S^{n}$ of Brouwer degree $k$.

Representing elements of $\pi_{n}\left(S^{n}\right)$ by polynomial maps is an old question [1, regarding Algebraic Geometry, Algebraic Topology and Differential Topology, which was affirmatively solved by Wood, in 1968, provided that $n$ is odd (theorem 1 of [2], see [3] as well for the complex sphere). Nevertheless, as far as I know, this problem is still open for $n$ even; our theorem settles it when the Brouwer degree is odd. In both cases, even and odd dimension, the polynomial maps constructed are homogeneous; therefore the problem of representing elements of $\pi_{n}\left(S^{n}\right)$ by homogeneous polynomial maps is solved now, since only zero and the odd topological degrees may be represented in this way when $n$ is even as any standard argument shows.

On the other hand the proof of theorem 1 of [3] makes use of a natural polynomial map of topological (or Brouwer) degree 2 (lemmas 4 and 5). Nothing similar is known for $n$ even; however the polynomial map $x \in \mathbb{R}^{3} \rightarrow\left(x_{1}^{2}-x_{2}^{2}, 2 x_{1} x_{2}, x_{3}\right) \in \mathbb{R}^{3}$ sends $S_{2}^{2}$ into $S^{2}$ with topological degree 2 . Part (b) of our theorem generalizes this map.

For proving Theorem 1 we start by constructing a family of real polynomials in one variable by means of an elementary Analysis reasoning. Let $\varphi_{\ell}=\sum_{j=0}^{\ell} a_{j} t^{j}$

Received by the editors May 11, 2006.

2000 Mathematics Subject Classification. Primary 55Q40; Secondary 57R19, 14P25.

The author thanks F. Gomez for pointing out to him this kind of problems.

(C)2007 American Mathematical Society Reverts to public domain 28 years from publication 
be the Taylor expansion of $\varphi=(1-t)^{-1 / 2}$, at zero, up to order $\ell$; that is to say, $a_{j}=\frac{(2 j-1)(2 j-3) \cdots 1}{2^{j} \cdot j !}$. Since the radius of convergence of the power series $\sum_{j=0}^{\infty} a_{j} t^{j}$ is 1 and each $a_{j}>0$, we have $a_{0}=1 \leq \varphi_{\ell} \leq(1-t)^{-1 / 2}, t \in[0,1)$, whence $(t-1) \varphi_{\ell}^{2}(t)+1 \geq 0$ and $\varphi_{\ell}(t) \geq 1$ when $t \geq 0$ (both inequalities are obvious if $t \geq 1)$.

On the other hand if we set $\varphi=t^{\ell+1} R+\varphi_{\ell}$, then $\sum_{j=0}^{\infty} t^{j}=(1-t)^{-1}=\varphi^{2}=$ $t^{\ell+1} \tilde{R}+\varphi_{\ell}^{2}$ on $(-1,1)$. Therefore $\varphi_{\ell}^{2}=t^{\ell+1} S+\sum_{j=0}^{\ell} t^{j}$ where $S$ is a polynomial in $t$. From that follows the existence of a polynomial $\lambda_{\ell}$ of degree $\ell$ such that $(t-1) \varphi_{\ell}^{2}(t)+1=t^{\ell+1} \lambda_{\ell}$.

Lemma 2. For every $\ell$ one has $(t-1) \varphi_{\ell}^{2}+1=t^{\ell+1} \lambda_{\ell}$ where $\lambda_{\ell}$ is a polynomial of degree $\ell$. Moreover $\lambda_{\ell}(t) \geq 0$ and $\varphi_{\ell}(t)>0$ for each $t \in \mathbb{R}$ if $\ell$ is even, and for any $t \geq 0$ if $\ell$ is odd.

Proof. It will suffice to show that $\lambda_{\ell}(t) \geq 0$ and $\varphi_{\ell}(t)>0$ if $\ell \geq 2$ is even and $t<0$. First we will prove, by induction on $\ell$, the existence of a $\delta_{\ell}>0$ such that $\varphi_{\ell}$ is strictly decreasing on $\left(-\infty,-1+\delta_{\ell}\right)$. Note that $\varphi_{\ell}=a_{\ell} t^{\ell}+a_{\ell-1} t^{\ell-1}+\varphi_{\ell-2}=$ $a\left((2 \ell-1) t^{\ell}+2 \ell t^{\ell-1}\right)+\varphi_{\ell-2}$ where $a>0$.

By the induction hypothesis or because $\varphi_{0}=1$, the polynomial $\varphi_{\ell-2}$ is decreasing on $\left(-\infty,-1+\delta_{\ell-2}\right)$, or on $\mathbb{R}$ if $\ell=2$. But the derivative $\left((2 \ell-1) t^{\ell}+2 \ell t^{\ell-1}\right)^{\prime}=$ $\left((2 \ell-1) \ell t^{\ell-1}+2 \ell(\ell-1) t^{\ell-2}\right)<0$ on $(-\infty,-1]$, so $\varphi_{\ell}$ is strictly decreasing on some interval $\left(-\infty,-1+\delta_{\ell}\right)$.

We show now that $\varphi_{\ell}(t)>(1-t)^{-1 / 2}>0$ if $t<0$. As $(1-t)^{-1 / 2}$ is strictly increasing, it is enough to prove our result on $(-1,0)$. On this interval $\lim _{\ell \rightarrow \infty}\left\{\varphi_{\ell}(t)\right\}=\sum_{j=0}^{\infty} a_{j} t^{j}=(1-t)^{-1 / 2}$. But the series $\sum_{j=0}^{\infty} a_{j} t^{j}$ is alternating, and the sequence $\left\{a_{j}|t|^{j}\right\}_{j \in \mathbb{N}}$, whose limit is zero, is strictly decreasing; then $\varphi_{\ell}(t)>(1-t)^{-1 / 2}>0$ for $\ell$ even.

Finally, if $\varphi_{\ell}(t)>(1-t)^{-1 / 2}>0$ for any $t<0$, a straightforward calculation shows that $(t-1) \varphi_{\ell}^{2}(t)+1<0$, whence $\lambda_{\ell}(t) \geq 0$ since $t^{\ell+1}<0$.

Recall that any polynomial $\mu$ which does not take negative values has even degree and can be written $\mu=\mu_{1}^{2}+\mu_{2}^{2}$, where $\mu_{1}$ and $\mu_{2}$ are polynomials of degree $\leq$ the half of degree of $\mu$. Therefore by setting $k=\ell+1, \alpha=\varphi_{\ell}, \lambda_{\ell}=\mu, \beta_{1}=\mu_{1}$ and $\beta_{2}=\mu_{2}$ one has:

Corollary 3. For any odd natural number $k$ there exist three polynomials $\alpha, \beta_{1}$, $\beta_{2}$, the first one of degree $k-1$ and the other two with degree $\leq \frac{k-1}{2}$, such that $\alpha(t)>0$ and $(1-t) \alpha^{2}(t)+t^{k}\left(\beta_{1}^{2}(t)+\beta_{2}^{2}(t)\right)=1$ anywhere.

Let us prove part (a) of Theorem 1. Since topological degrees \pm 1 may be represented by linear maps, we can assume $k \geq 1$. On $\mathbb{C} \times \mathbb{R}^{n-1}=\mathbb{R}^{n+1}$, endowed with coordinates $(z, y)=\left(z, y_{1}, \ldots, y_{n-1}\right)$ for which $S^{n}=\left\{(z, y) ;|z|^{2}+y_{1}^{2}+\ldots+y_{n-1}^{2}=1\right\}$, we define

$$
F(z, y)=\left(\left(\beta_{1}\left(|z|^{2}\right)+i \beta_{2}\left(|z|^{2}\right)\right) z^{k}, \alpha\left(|z|^{2}\right) y\right)
$$

where $\alpha, \beta_{1}$ and $\beta_{2}$ are as in Corollary 3. Then $F\left(S^{n}\right) \subset S^{n}$.

Set $S^{1}=\left\{(z, 0) ;|z|^{2}=1\right\} \subset S^{n}$. As $\alpha(t)>0$ for each $t \in \mathbb{R}, F^{-1}\left(S^{1}\right)=S^{1}$ and $F$ preserves the orientation transversely to $S^{1}$. Hence the maps $F_{\mid S^{1}}$ and $F_{\mid S^{n}}$ have the same topological degree, that is to say, $k$ (consider a regular value belonging to $\left.S^{1}\right)$. 
By construction all the monomials of $F$ have odd degree $\leq 2 k-1$. Multiplying all of them by a suitable power of $|z|^{2}+y_{1}^{2}+\ldots+y_{n-1}^{2}$ the map $F$ becomes homogeneous of algebraic degree $2 k-1$, whereas $F_{\mid S^{n}}$ does not change.

For proving (b), first we set $\tilde{\lambda}_{\ell}(t)=\lambda_{\ell}\left(t^{2}\right)$ and $\tilde{\varphi}_{\ell}(t)=\varphi_{\ell}\left(t^{2}\right)$. By Lemma 2 we have $\left(t^{2}-1\right) \tilde{\varphi}_{\ell}^{2}(t)+1=t^{2 \ell+2} \tilde{\lambda}_{\ell}(t), \tilde{\varphi}_{\ell}(t)>0$ and $\tilde{\lambda}_{\ell}(t) \geq 0$ for any $t \in \mathbb{R}$. This allows us to find, for every natural number $\tilde{k} \geq 1$, three polynomials $\tilde{\alpha}, \tilde{\beta}_{1}, \tilde{\beta}_{2}$ such that $\tilde{\alpha}(t)>0$ and $\left(1-t^{2}\right) \tilde{\alpha}^{2}(t)+t^{2 \tilde{k}}\left(\tilde{\beta}_{1}^{2}(t)+\tilde{\beta}_{2}^{2}(t)\right)=1$ anywhere.

Consider on $\mathbb{R}^{n+1}=\mathbb{R}^{2 r} \times \mathbb{R}^{n-2 r+1}$ coordinates $(x, y)=\left(x_{1}, \ldots, x_{2 r}, y_{1}, \ldots\right.$, $\left.y_{n-2 r+1}\right)$. Let $f: \mathbb{R}^{2 r} \rightarrow \mathbb{R}^{2 r}$ be a homogeneous polynomial map of algebraic degree $2 \tilde{k}$, sending $S^{2 r-1}$ into $S^{2 r-1}$ with topological degree $k= \pm 2 \tilde{k}$, which always exists (see [3]) and $J: \mathbb{R}^{2 r} \rightarrow \mathbb{R}^{2 r}$ the isomorphism given by $J x=\left(-x_{2}, x_{1}, \ldots,-x_{2 r}, x_{2 r-1}\right)$, that is to say, the canonical complex structure of $\mathbb{R}^{2 r}$. One defines (if $\tilde{k}=0$ just consider a constant map):

$$
\left.F(z, y)=\left(\tilde{\beta}_{1}\left(\|x\|^{2}\right) f(x)+\tilde{\beta}_{2}(\|x\|)^{2}\right) J f(x), \tilde{\alpha}\left(\|x\|^{2}\right) y\right) .
$$

Then $F\left(S_{2 r}^{n}\right) \subset S^{n}$ and the same argument as in part (a), applied to $S^{2 r-1}=$ $\left\{(x, 0) ;\|x\|^{2}=1\right\} \subset S_{2 r}^{n}$, shows that the topological degree of $F: S_{2 r}^{n} \rightarrow S^{n}$ equals $k$.

\section{REFERENCES}

1. P. F. Baum, Quadratic maps and stable homotopy groups of spheres, Illinois J. Math. 11 (1967), 586-595. MR0220285 (36:3351)

2. R. Wood, Polynomial maps from spheres to spheres, Invent. Math. 5 (1968), 163-168. MR0227999 (37:3583)

3. R. Wood, Polynomial maps of affine quadrics, Bull. London Math. Soc. 25 (1993), 491-497. MR.1233414 (94i:55019)

Geometría y Topología, Facultad de Ciencias, Campus de Teatinos, 29071 Málaga, SPAIN

Current address: Geometría y Topología, Facultad de Ciencias, Campus de Teatinos, 29071 Málaga, Spain

E-mail address: turiel@agt.cie.uma.es 